Bull. Soc. math. France

132 (4), 2004, p. 591-612

\title{
ON MEROMORPHIC FUNCTIONS DEFINED BY A DIFFERENTIAL SYSTEM OF ORDER 1
}

\author{
By TRISTAN TORRELli
}

\begin{abstract}
Given a germ $h$ of holomorphic function on $\left(\mathbb{C}^{n}, 0\right)$, we study the condition: "the ideal $A n_{\mathcal{D}} 1 / h$ is generated by operators of order 1 ". We obtain here full characterizations in the particular cases of Koszul-free germs and unreduced germs of plane curves. Moreover, we prove that this condition holds for a special type of hyperplane arrangements. These results allow us to link this condition to the comparison of de Rham complexes associated with $h$.

RÉSUMÉ (Sur les germes de fonctions méromorphes définis par un système différentiel d'ordre 1)

Étant donné un germe de fonction holomorphe $h$ défini au voisinage de l'origine de $\mathbb{C}^{n}$, nous étudions la condition : «l'idéal $\mathrm{Ann}_{\mathcal{D}} 1 / h$ est engendré par des opérateurs d'ordre $1 »$. Nous obtenons ici des caractérisations complètes dans le cas des germes Koszul-libres et dans celui des germes de courbes planes non réduits. De plus, nous montrons que cette condition est vérifiée pour un type particulier d'arrangements d'hyperplans. Ces résultats nous permettent de relier cette condition à la comparaison de complexes de de Rham associés à $h$.
\end{abstract}

Texte reçu le 24 février 2003, révisé le 29 septembre 2003, accepté le 14 novembre 2003 Tristan Torrelli, Université Henri Poincaré Nancy 1, Institut Élie Cartan, UMR 7502 CNRS - INRIA - UHP, B.P. 239, 54506 Vandoeuvre-lès-Nancy Cedex (France)

E-mail : torrelli@math.unice.fr • Url : http://www.iecn.u-nancy.fr/

2000 Mathematics Subject Classification. - 32C38, 32S25, 14F10, 14F40.

Key words and phrases. - Germs of meromorphic functions, $\mathcal{D}$-modules, free divisors, arrangements of hyperplanes, logarithmic de Rham complex, logarithmic comparison theorem. I thank the support of the P.A.I. 02577 RG Systèmes différentiels et singularités. 


\section{Introduction}

Let $h \in \mathcal{O}=\mathbb{C}\left\{x_{1}, \ldots, x_{n}\right\}$ be a nonzero germ of holomorphic function such that $h(0)=0$. We denote by $\mathcal{O}[1 / h]$ the ring $\mathcal{O}$ localized by the powers of $h$. Let $\mathcal{D}=\mathcal{O}\left\langle\partial / \partial x_{1}, \ldots, \partial / \partial x_{n}\right\rangle$ be the ring of linear differential operators with holomorphic coefficients and $F \cdot \mathcal{D}$ its filtration by order. As usual, we identify $\operatorname{gr}^{F} \mathcal{D}$ with the polynomial ring $\mathcal{O}[\xi]=\mathcal{O}\left[\xi_{1}, \ldots, \xi_{n}\right]$.

Given $a / h^{\ell} \in \mathcal{O}[1 / h]$ nonzero, we consider the following condition:

The left ideal $\operatorname{Ann}_{\mathcal{D}} a / h^{\ell} \subset \mathcal{D}$ of operators annihilating $a / h^{\ell}$ is generated by operators of order 1 .

This condition appears when studying the elements of the holonomic $\mathcal{D}$-modules $\mathcal{O}[1 / h]$ and $\mathcal{O}[1 / h] / \mathcal{O}$ (see [18]). Moreover, it is directly linked to the so-called "Logarithmic Comparison Theorem" (see below). The aim of this work is to explicit this condition. First we remark the following fact.

Proposition 1.1. - Let $a, h \in \mathcal{O}$ be germs of holomorphic functions without common factor. If the ideal $\operatorname{Ann}_{\mathcal{D}} a / h$ is generated by operators of order 1 , then $a$ is a unit.

So, without loss of generality, we will suppose that $a=1$. When $h$ defines a hypersurface with isolated singularity, we have obtained in [18] the following characterization.

THEOREM 1.2. - Let $h \in \mathcal{O}$ be a germ of a holomorphic function defining an isolated singularity. Let $\ell \in \mathbb{N}^{*}$ be a nonnegative integer. Then the ideal $\operatorname{Ann}_{\mathcal{D}} 1 / h^{\ell}$ is generated by operators of order 1 if and only if the following conditions are verified:

(a) the germ $h$ is weighted-homogeneous,

(b) the smallest integral root of the Bernstein polynomial of $h$ is strictly greater than $-\ell-1$.

We recall that a nonzero germ $h$ is weighted-homogeneous of weight $d \in \mathbb{Q}^{+}$ for a system $\alpha \in\left(\mathbb{Q}^{*+}\right)^{n}$ if there exists a system of coordinates in which $h$ is a linear combination of monomials $x_{1}^{\gamma_{1}} \cdots x_{n}^{\gamma_{n}}$ with $\sum_{i=1}^{n} \alpha_{i} \gamma_{i}=d$. Moreover, the condition (b) means that $1 / h^{\ell}$ generates the $\mathcal{D}$-module $\mathcal{O}[1 / h]$ (see [11, Prop. 6.2] and [2, Prop. 6.1.18, 6.3.15 \& 6.3.16]; for the definition of the Bernstein polynomial, see the beginning of part 2).

What does remain true without any assumption on $h$ ? First of all, the condition (b) is always necessary.

Proposition 1.3. - Let $h \in \mathcal{O}$ be a nonzero germ of holomorphic function with $h(0)=0$. Let $\ell \in \mathbb{N}^{*}$ be a nonnegative integer such that the ideal $\operatorname{Ann}_{\mathcal{D}} 1 / h^{\ell}$ is generated by operators of order 1 . Then the smallest integral root of the Bernstein polynomial of $h$ is strictly greater than $-\ell-1$.

TOME $132-2004-\mathrm{N}^{\mathrm{O}} 4$ 
On the other hand, $h$ is not always weighted-homogeneous (Example 1.5). So, let us denote the condition:

$\left(\mathrm{a}^{\prime}\right) h$ belongs to the ideal of its partial derivatives.

In other words, there exists a vector field $v \in \mathcal{D}$ such that $v(h)=h$, and we will say that $h$ is Euler-homogeneous. In the case of hypersurfaces with isolated singularities, K. Saito has proved that these two conditions coincide (see [13]). We conjecture the following fact.

CONJECTURE 1.4. - If there exists a nonnegative integer $\ell \in \mathbb{N}^{*}$ such that $\mathrm{Ann}_{\mathcal{D}} 1 / h^{\ell}$ is generated by some operators of order 1 , then $h$ is Eulerhomogeneous.

Reciprocally, conditions $\left(\mathrm{a}^{\prime}\right)$ and (b) are not always enough to have $A n_{\mathcal{D}} 1 / h^{\ell}$ generated by operators of order 1 (see Example 1.9). Nevertheless, they are sufficient when the ideal $\mathrm{Ann}_{\mathcal{D}} h^{s}$ is generated by operators of order 1 (this is true in the case of isolated singularities (see [12, p. 117], or [23, Thm 2.19])). Indeed, if $h$ is Euler-homogeneous, then we have a decomposition:

$$
\operatorname{Ann}_{\mathcal{D}[s]} h^{s}=\mathcal{D}[s](s-v)+\mathcal{D}[s] \operatorname{Ann}_{\mathcal{D}} h^{s}
$$

moreover, with the condition (b), $\operatorname{Ann}_{\mathcal{D}} 1 / h^{\ell}$ is obtained by fixing $s=-\ell$ in a system of generators of $\operatorname{Ann}_{\mathcal{D}[s]} h^{s}$ (see [18, Prop. 3.1]). Finally, the fact that $\operatorname{Ann}_{\mathcal{D}} 1 / h^{\ell}$ is generated by operators of order 1 does not imply that so is $\operatorname{Ann}_{\mathcal{D}} h^{s}$.

EXAMPLE 1.5 (see [3], [4], [6]). - Let $h=x_{1} x_{2}\left(x_{1}+x_{2}\right)\left(x_{1}+x_{2} x_{3}\right)$. It is an Euler-homogeneous polynomial which is not weighted-homogeneous. Indeed, if there exists a change of coordinates $\varphi=\left(\varphi_{1}, \varphi_{2}, \varphi_{3}\right)$ - with $\varphi(0)=0$ - such that $h \circ \varphi$ is a weighted-homogeneous polynomial for $\alpha \in\left(\mathbb{Q}^{*+}\right)^{3}$, then its factors are weighted-homogeneous too. Thus the polynomials $\varphi_{1}, \varphi_{2}$ and $\varphi_{2} \varphi_{3}$ must have the same weight, and this is absurd.

The ideal $\mathrm{Ann}_{\mathcal{D}} 1 / h$ is generated by the operators:

$$
\begin{aligned}
& S_{1}=\left(x_{1}+x_{2} x_{3}\right) \frac{\partial}{\partial x_{3}}+x_{2}, \\
& S_{2}=x_{2}\left(x_{1}+x_{2}\right) \frac{\partial}{\partial x_{2}}-x_{1}\left(x_{3}-1\right) \frac{\partial}{\partial x_{3}}+x_{1}+3 x_{2}, \\
& S_{3}=x_{1} \frac{\partial}{\partial x_{1}}+x_{2} \frac{\partial}{\partial x_{2}}+4 .
\end{aligned}
$$

The $\mathcal{O}$-module $\operatorname{Ann}_{\mathcal{D}} h^{s} \cap F_{1} \mathcal{D}$ is generated by:

$$
Q_{1}=4 S_{1}-x_{2} S_{3}, \quad Q_{2}=4 S_{2}-\left(x_{1}+3 x_{2}\right) S_{3}
$$

BULletin DE LA SOCiÉtÉ MATHÉMATIQUE DE FRANCE 
and it defines an ideal $I \subset \mathcal{D}$ which does not coincide with $\operatorname{Ann}_{\mathcal{D}} h^{s}$. Indeed, one can verify that the following operator:

$$
\begin{aligned}
P=2 x_{2}^{2} & \frac{\partial}{\partial x_{1}} \frac{\partial}{\partial x_{2}}-2 x_{2}^{2} \frac{\partial^{2}}{\partial x_{2}}-2\left(x_{1}+3 x_{2}\right) x_{3} \frac{\partial}{\partial x_{1}} \frac{\partial}{\partial x_{3}} \\
+ & 2\left(x_{1}-2 x_{2}+5 x_{2} x_{3}\right) \frac{\partial}{\partial x_{2}} \frac{\partial}{\partial x_{3}}+8\left(1-x_{3}\right) x_{3} \frac{\partial^{2}}{\partial x_{3}} \\
& -x_{1} \frac{\partial}{\partial x_{1}}-x_{2} \frac{\partial}{\partial x_{2}}-4\left(2 x_{3}+1\right) \frac{\partial}{\partial x_{3}}
\end{aligned}
$$

annihilates $h^{s}$. But $P$ does not belong to $I$ because the ideal $\operatorname{gr}^{F} I$ is generated by the principal symbols $\sigma\left(Q_{1}\right), \sigma\left(Q_{2}\right)$, and in particular $\operatorname{gr}^{F} I \subset\left(x_{1}, x_{2}\right) \mathcal{O}[\xi]$ even if $\sigma(P) \notin\left(x_{1}, x_{2}\right) \mathcal{O}[\xi]$.

In the two following parts, we try to extend to other situations the characterization given by Theorem 1.2. We begin with the case of plane curves.

TheOREM 1.6. - Let $h \in \mathbb{C}\left\{x_{1}, x_{2}\right\}$ be nonzero with $h(0)=0$, and let $\ell \in \mathbb{N}^{*}$ be a nonnegative integer.

(i) The ideal $\operatorname{Ann}_{\mathcal{D}} 1 / h^{\ell}$ is generated by operators of order 1 if and only if $h$ is weighted-homogeneous.

(ii) Let $N \in \mathbb{N}^{*}$ be a nonnegative integer greater than or equal to 2 . Let $\tilde{b}(s) \in$ $\mathbb{C}[s]$ be the reduced Bernstein polynomial of $h$. Then the ideal $\operatorname{Ann}_{\mathcal{D}} 1 /\left(h+x_{3}^{N}\right)^{\ell}$ is generated by operators of order 1 if and only if the following conditions are verified:

(a) the germ $h$ is weighted-homogeneous,

(b) $\ell \geq 2$, or $\ell=1$ and -2 is not a root of a polynomial $\tilde{b}(s+i / N)$, for $1 \leq i \leq N-1$.

If $h$ is reduced, it is a very particular case of Theorem 1.2 (for another proof of (i), see [6]). We use that the Euler-homogeneous germs of plane curves are weighted-homogeneous (Proposition 3.4), which comes from K. Saito ([13]).

Another part is devoted to a variant of Theorem 1.2, where the assumption on $h$ is replaced by a condition on the graded ideal of $\mathrm{Ann}_{\mathcal{D}} 1 / h^{\ell}$.

THEOREM 1.7. - Let $h \in \mathcal{O}$ be a nonzero germ such that $h(0)=0$, and $\ell \in \mathbb{N}^{*}$. Suppose that the $\mathcal{O}$-module $\mathrm{Ann}_{\mathcal{D}} 1 / h^{\ell} \cap F_{1} \mathcal{D}$ is generated by operators $Q_{1}, \ldots, Q_{w}$ such that: $\operatorname{gr}^{F} \mathcal{D}\left(Q_{1}, \ldots, Q_{w}\right)=\left(\sigma\left(Q_{1}\right), \ldots, \sigma\left(Q_{w}\right)\right) \operatorname{gr}^{F} \mathcal{D}$. Then the ideal $\operatorname{Ann}_{\mathcal{D}} 1 / h^{\ell}$ is generated by a system of operators of order 1 if and only if the following conditions are verified:

(a) the germ $h$ belongs to the ideal of its partial derivatives,

(b) the smallest integral root of the Bernstein polynomial of $h$ is strictly greater than $-\ell-1$,

(c) the ideal $\operatorname{Ann}_{\mathcal{D}} h^{s}$ is generated by operators of order 1.

TOME $132-2004-\mathrm{N}^{\mathrm{O}} 4$ 
Moreover, $\operatorname{Ann}_{\mathcal{D}} h^{s}$ is also generated by $Q_{j}(1) Q_{i}-Q_{i}(1) Q_{j}, 1 \leq i \leq w, i \neq j$, where $j$ is such that $Q_{j}(1)$ is a unit.

It is not easy to find a family of germs which verify this assumption. Except for the case of weighted-homogeneous isolated singularities (see [19, Prop. 4.3]), one can prove that it is also verified for a particular type of free germs - in the sense of K. Saito [14]: the so-called Koszul-free germs.

Recall that a reduced germ $h \in \mathcal{O}$ is free if the $\mathcal{O}$-module $\operatorname{Der}(\log h) \subset \mathcal{D}$ of vector fields $v$ such that $v(h) \in h \mathcal{O}$ is free (its rank is also equal to $n$ ). The germ $h$ is said to be Koszul-free if there exists a basis $\left\{\delta_{1}, \ldots, \delta_{n}\right\}$ of $\operatorname{Der}(\log h)$ such that the sequence of principal symbols $\left(\sigma\left(\delta_{1}\right), \ldots, \sigma\left(\delta_{n}\right)\right)$ is $\operatorname{gr}^{F} \mathcal{D}$-regular (see [3]). For example, germs of reduced plane curves and locally weightedhomogeneous free germs are Koszul-free (see [14, Cor. 1.7] and [4]).

Corollary 1.8. - Let $h \in \mathcal{O}$ be a Koszul-free germ. Then the ideal $\operatorname{Ann}_{\mathcal{D}} 1 / h$ is generated by operators of order 1 if and only if the following conditions are verified:

(a) the germ $h$ is Euler-homogeneous,

(b) -1 is the only integral root of the Bernstein polynomial of $h$,

(c) the ideal $\operatorname{Ann}_{\mathcal{D}} h^{s}$ is generated by operators of order 1.

Suppose furthermore that $h$ is Euler-homogeneous. Let $\left\{\delta_{1}, \ldots, \delta_{n}\right\}$ be a basis of $\operatorname{Der}(\log h)$ such that $\delta_{1}(h)=h$ and $\delta_{i}(h)=0,2 \leq i \leq n$. Then condition (c) is equivalent to:

$\left(\mathrm{c}^{\prime}\right)$ the sequence $\left(h, \sigma\left(\delta_{2}\right), \ldots, \sigma\left(\delta_{n}\right)\right)$ is $\operatorname{gr}^{F} \mathcal{D}$-regular.

The following example shows that condition (c) is neither a consequence of the assumption of Theorem 1.7 on $\operatorname{gr}^{F} \mathrm{Ann}_{\mathcal{D}} 1 / h^{\ell}$ nor a consequence of conditions (a) and (b) for a Koszul-free germ.

EXAMPLE 1.9. - Let $\tilde{h}=x_{1}^{5}+x_{2}^{5}+x_{1}^{2} x_{2}^{2}$. It is a Koszul-free germ which is not Euler-homogeneous. Let $h=\exp \left(x_{3}\right) \tilde{h}$. Using Saito criterion (see [14]), it is easy to see that the Euler-homogeneous germ $h$ is Koszul-free. Up to a unit, $h$ and $\tilde{h}$ are equal ; so they have the same Bernstein polynomial. In particular, -1 is the only integral root of the Bernstein polynomial of $h$. So $h$ verifies conditions (a) and (b), but not (c). Indeed, condition " $A n_{\mathcal{D}} 1 / h$ is generated by operators of order 1 " only depends on the hypersurface germ defined by $h$, and it is not verified by $\tilde{h}$ (see Theorem 1.6).

Let us remark that this characterization can not be extented to the case of free germs (since the germ of Example 1.5 is free).

In the last part, we study the case of a hyperplane arrangement defined by $h=0$ in $\mathbb{C}^{n}$. Indeed, A. Leykin has proved the following fact.

BULLETIN DE LA SOCIÉtÉ MATHÉMATIQUE DE FRANCE 
Proposition 1.10 ([21, Thm 5.1]). - The Bernstein polynomial of any arrangement has only -1 as integral root.

Is the ideal $A_{n} n_{\mathcal{D}} 1 / h$ generated by operators of order 1 ? We prove here that it is true for the union of a generic hyperplane arrangement with a hyperbolic arrangement (Theorem 5.2). Moreover, our geometric proof gives an explicit system of generators of $\mathrm{Ann}_{\mathcal{D}} 1 / h$. In the particular case of a generic central hyperplane arrangement (Corollary 5.3), this answers a conjecture of U. Walther [21].

We end this introduction by linking these results to the Logarithmic Comparison Theorem. For any hypersurface $D \subset \mathbb{C}^{n}$, we denote by $\Omega^{\bullet}(\star D)$ the de Rham complex of differential meromorphic forms with poles along $D$ and by $\Omega \bullet(\log D)$ its subcomplex of logarithmic forms, introduced by K. Saito ([14]). One says that the Logarithmic Comparison Theorem (LCT) holds for $D$ if the inclusion:

$$
i_{D}: \Omega^{\bullet}(\log D) \hookrightarrow \Omega^{\bullet}(\star D)
$$

is a quasi-isomorphism. Indeed, according to Grothendieck Comparison Theorem (see [9]), the complex $\Omega^{\bullet}(\log D)$ computes also the cohomology of the complementary of $D \subset \mathbb{C}^{n}$. So, it is natural to search for conditions on $D$ such that the LCT holds for $D$. For instance, F.J. Castro-Jiménez, D. Mond and L. Narváez-Macarro have proved that it is true for all locally weightedhomogeneous free divisors (i.e. free and weighted-homogeneous at all their points) (see [5]). We conjecture that the following fact is always true.

Conjecture 1.11. - Let $h_{D} \in \mathcal{O}$ be a reduced equation of $(D, 0)$. Then the ideal $\mathrm{Ann}_{\mathcal{D}} 1 / h_{D}$ is generated by operators of order 1 if and only if the Logarithmic Comparison Theorem holds for $(D, 0)$.

Let us give now three significant results at the origin of this assertion. First, using F.J. Calderón-Moreno works on differential logarithmic operators relative to a free divisor (see [3]), F.J. Castro-Jiménez and J.M. Ucha-Enríquez have proved that for a locally weighted-homogeneous free divisor, the de Rham complex of the holonomic $\mathcal{D}$-Module: $\widetilde{M}^{\log }=\mathcal{D} / \widetilde{I}^{\log }$, where $\widetilde{I}^{\log }$ is the left ideal generated by $\operatorname{Ann}_{\mathcal{D}} 1 / h_{D} \cap F_{1} \mathcal{D}$, is quasi-isomorphic to $\Omega \bullet(\log D)([7])$. Moreover, using the de Rham functor, the morphism:

$$
\phi_{D}: \widetilde{M}^{\log } \longrightarrow \mathcal{O}(\star D), \quad P+\widetilde{I}^{\log } \longmapsto P \cdot \frac{1}{h_{D}}
$$

is an isomorphism if and only if the morphism $i_{D}$ of $(1)$ is a quasi-isomorphism. The same result for a Koszul-free divisor is announced by L. Narváez-Macarro.

But, from Proposition 1.3 and diagram $\widetilde{M}^{\log } \rightarrow \mathcal{D} 1 / h_{D} \hookrightarrow \mathcal{O}(\star D)$, it is clear that $\phi_{D}$ is an isomorphism if and only if $A n_{\mathcal{D}} 1 / h_{D}$ is generated by operators of order 1. In particular, Corollary 1.8 gives a characterization of the LCT in the case of Koszul-free germs.

TOME $132-2004-\mathrm{N}^{\mathrm{O}} 4$ 
Moreover, M. Holland and D. Mond have obtained some characterizations of the LCT for weighted-homogeneous hypersurface with isolated singularity (see [10]). In terms of weight of elements of a weighted-homogeneous co-basis $E$ of the jacobian ideal of $h_{D}$, they have obtained the following condition: there is no vector $e \in E$ whose weight belongs to the set $\{i \varrho-|\alpha| ; 1 \leq i \leq n-2\}$ (where $\alpha \in\left(\mathbb{Q}^{*+}\right)^{n}$ is the weight system and $\varrho \in \mathbb{Q}^{*+}$ is the weight of $h_{D}$ ). Using the formula of Bernstein polynomial of a weighted-homogeneous isolated singularity (see $[23, \S 11]$ ), it is also easy to check that this is equivalent to the fact that -1 is the only integral root of the Bernstein polynomial of $h_{D}$. So, from Theorem 1.2, our conjecture is verified.

Finally, H. Terao and S. Yuzvinsky conjecture that the LCT holds for any central hyperplane arrangements in $\mathbb{C}^{n}$. They have proved it when $n \leq 5$, and for special types of arrangement (see [22]). So, Theorem 5.2 agrees with our assertion.

\section{Two necessary conditions}

In this part, we prove Propositions 1.1 and 1.3. First, we recall some elementary facts about Bernstein polynomials.

Given a nonzero germ of holomorphic function $f \in \mathcal{O}$, there exists functional equations:

$$
b(s) f^{s}=P(s) f^{s+1}
$$

in $\mathcal{O}[1 / f, s] f^{s}$, where $b(s) \in \mathbb{C}[s]$ and $P(s) \in \mathcal{D}[s]=\mathcal{D} \otimes \mathbb{C}[s]$ are nonzero (see [11]). The Bernstein polynomial of $f$ at the origin is the unitary polynomial $b(s)$ of smallest degree which verifies such an identity. When $f$ is not a unit, it is easy to remark that -1 is a root of $b(s)$. So, we call the reduced Bernstein polynomial, denoted by $\tilde{b}(s)$, the quotient of $b(s)$ by $s+1$.

The proof of Proposition 1.1 uses the following fact.

Proposition 2.1. - Let $a \in \mathcal{O}$ be a nonzero germ. Then $a$ is a unit if and only if the annihilator in $\mathcal{D}$ of $a, \operatorname{Ann}_{\mathcal{D}} a$, is generated by operators of order 1 .

Proof. - If $a$ is a unit, it is obvious that $\mathrm{Ann}_{\mathcal{D}} a$ is generated by the operators $\left(\partial / \partial x_{i}\right) a^{-1}, 1 \leq i \leq n$. Now, let us suppose that $\operatorname{Ann}_{\mathcal{D}} a$ is generated by $Q_{1}, \ldots, Q_{w} \in F_{1} \mathcal{D}$ and that $a$ is not a unit. Thus $s+1$ is a factor of the Bernstein polynomial of $a$, denoted by $b(s)$.

Using a Bernstein equation of $a$, we get:

$$
b(s) b(s+1) a^{s}=P(s) a^{s+2}
$$

BULletin DE LA SOCiÉtÉ MATHÉMATIQUE DE FRANCE 
where $P(s) \in \mathcal{D}[s]$. As -1 is a root of $b(s)$, the operator $P(-1)$ annihilates $a$. So it may be written $P(-1)=\sum_{i=1}^{w} A_{i} Q_{i}$ with $A_{i} \in \mathcal{D}$. Thus, we have:

$$
P(s)=(s+1) P^{\prime}(s)+\sum_{i=1}^{w} A_{i} Q_{i},
$$

with $P^{\prime}(s) \in \mathcal{D}[s]$, and the previous identity becomes:

$$
b(s) b(s+1) a^{s}=(s+1)\left[P^{\prime}(s) a+\sum_{i=1}^{w} A_{i}\left[Q_{i}, a\right]\right] a^{s+1} .
$$

By division by $s+1$, we deduce that $\tilde{b}(s) b(s+1)$ is a multiple of the Bernstein polynomial of $a$. But this is absurd: the multiplicity of the root -1 in $b(s)$ is strictly greater than the one of $\tilde{b}(s) b(s+1)$ because $b(0) \neq 0$ (the roots of the Bernstein polynomial of any germ are strictly negative, see [11]). Hence, $a$ must be a unit.

Proof of Proposition 1.1. - If $h$ is a unit, the assertion is a direct consequence of the previous result. So, we will suppose that $h(0)=0$. Let $\tilde{a}, \tilde{h}$ be holomorphic functions which define $a, h$ on a neighborhood $U \subset \mathbb{C}^{n}$ of the origin. Up to a restriction of $U$, we may assume that the zero set $V(\tilde{a}, \tilde{h}) \subset U$ has codimension 2 , and that the annihilator of $\tilde{a} / \tilde{h}$ is generated by operators on $U$ of order 1 . Then, at any point $M \in U$ such that $\tilde{h}(M) \neq 0$, the annihilator of $\tilde{a}$ verifies the same property, and from the previous proposition, we have $\tilde{a}(M) \neq 0$. Thus, $\tilde{a}$ has no zero in the complementary of $V(\tilde{a}, \tilde{h}) \subset U$, and then no zero at all in $U$. Hence, the germ $a$ is a unit.

Finally, let us give the proof of Proposition 1.3.

Proof. - Let $h \in \mathcal{O}$ be not a unit, and $\ell \in \mathbb{N}^{*}$ such that $\operatorname{Ann}_{\mathcal{D}} 1 / h^{\ell}$ is generated by operators $Q_{1}, \ldots, Q_{w}$ of order 1 . For $1 \leq i \leq w$, we denote by $q_{i}$ the germ $Q_{i}(1) \in \mathcal{O}$ and by $Q_{i}^{\prime} \in \mathcal{D}$ the vector field $Q_{i}-q_{i}$; thus we have $\ell Q_{i}^{\prime}(h)=q_{i} h$, $1 \leq i \leq w$. Let us suppose that the Bernstein polynomial of $h$, denoted $b(s)$, has an integral root strictly smaller than $-\ell$. We denote by $k \in \mathbb{Z}-\mathbb{N}$, the greatest root of $b(s)$ verifying this condition. Using a Bernstein equation which gives $b(s)$, we get:

$$
b(s) \cdots b(s-\ell-k-1) h^{s}=P(s) h^{s-\ell-k}
$$

where $P(s) \in \mathcal{D}[s]$. Thus $P(k)$ annihilates $h^{-\ell}$, and so, it may be written $P(k)=\sum_{i=1}^{w} A_{i} Q_{i}$ with $A_{i} \in \mathcal{D}, 1 \leq i \leq w$. If $P^{\prime}(s) \in \mathcal{D}[s]$ is the quotient of $P(s)$ by $s-k$, the previous equation becomes:

$$
\underbrace{b(s) \cdots b(s-\ell-k-1)}_{c(s)} h^{s}=(s-k)\left[P^{\prime}(s)+\frac{1}{\ell} \sum_{i=1}^{w} A_{i} q_{i}\right] h^{-\ell-k-1} \cdot h^{s+1}
$$

TOME $132-2004-\mathrm{N}^{\mathrm{O}} 4$ 
where $-\ell-k-1 \geq 0$ and the multiplicity of $k$ in $c(s)$ is the same in $b(s)$. Then, by division by $s-k$, we get a functional equation of the form (2) such that the polynomial in the left member is not a multiple of $b(s)$. But this is not possible, because $b(s)$ is the Bernstein polynomial of $h$. Hence we have the result.

\section{The case of plane curves}

The aim of this part is the proof of Theorem 1.6, which extends to the case of non reduced planes curves the characterization given by Theorem 1.2. First, we recall some results of K. Saito on the weakly weighted-homogeneous power series and the formal differential operators of order 1 (see [13]).

3.1. Normal form of formal differential operators of order 1 . - Let

$$
D=a_{1} \frac{\partial}{\partial x_{1}}+\cdots+a_{n} \frac{\partial}{\partial x_{n}}
$$

be a formal differential operator with $a_{i}(0)=0,1 \leq i \leq n$. We denote $\partial D / \partial x$ the jacobian matrix of $\left(a_{1}, \ldots, a_{n}\right)$. The operator $D$ is semi-simple in the coordinates $x_{1}, \ldots, x_{n}$ if $\partial D / \partial x$ is a diagonal matrix. The operator $D$ is nilpotent if the eigenvalues of $(\partial D / \partial x)_{\left.\right|_{0}}$ are zero.

Proposition 3.1. - Let $D=a_{1}\left(\partial / \partial x_{1}\right)+\cdots+a_{n}\left(\partial / \partial x_{n}\right)$ be a formal differential operator with $a_{i}(0)=0,1 \leq i \leq n$. Then there exist coordinates such that $D$ is a sum $D=D_{S}+D_{N}$ where $D_{S}$ is a semi-simple operator, $D_{N}$ is a nilpotent operator and $D_{S} D_{N}=D_{N} D_{S}$.

Proposition 3.2. - Let $D=a_{1}\left(\partial / \partial x_{1}\right)+\cdots+a_{n}\left(\partial / \partial x_{n}\right)$, be a formal differential operator with $a_{i}(0)=0,1 \leq i \leq n$. Let $D=D_{S}+D_{N}$ be its normal form in the coordinates $x_{1}, \ldots, x_{n}$. Let $f \in \mathbb{C}\left[\left[x_{1}, \ldots, x_{n}\right]\right]$ and $\lambda \in \mathbb{C}$. Then $D f=\lambda f$ if and only if $D_{S} f=\lambda f$ and $D_{N} f=0$.

Definition 3.3. - A formal power series $f \in \mathbb{C}\left[\left[x_{1}, \ldots, x_{n}\right]\right]$ is weakly weighted-homogeneous of type $\left(\alpha_{0}, \alpha_{1}, \ldots, \alpha_{n}\right) \in \mathbb{C}^{n+1}$ if we have:

$$
\alpha_{1} \gamma_{1}+\cdots+\alpha_{n} \gamma_{n}=\alpha_{0}
$$

for all monomial $x^{\gamma}$ which appears with a nonzero coefficient in the power expansion of $f$.

In other words, $f$ is an eigenfunction of the operator $\sum_{i=1}^{n} \alpha_{i} x_{i}\left(\partial / \partial x_{i}\right)$ for the eigenvalue $\alpha_{0}$.

From the previous propositions, we get the following result which is specific to dimension 2.

Proposition 3.4. - Let $h \in \mathbb{C}\left\{x_{1}, x_{2}\right\}$ be a nonzero germ such that $h(0)=0$. The following conditions are equivalent: 
1) the germ $h$ is weighted-homogeneous,

2) the germ $h$ is Euler-homogeneous,

3) there exists a formal change of coordinates $\phi$ such that $h \circ \phi$ is a weakly weighted-homogeneous power series.

Proof. - The implication 1) $\Rightarrow 2$ ) is clear. Let us prove 2$) \Rightarrow 3$ ). If $h$ is Eulerhomogeneous, there exists $D=a_{1}\left(\partial / \partial x_{1}\right)+a_{2}\left(\partial / \partial x_{2}\right) \in \mathcal{D}$ such that $D h=h$. If $a_{1}$ and $a_{2}$ are not units, the previous proposition implies the result. Otherwise, up to a change of coordinates, we may assume that $a_{1}=1, a_{2}=0$, and then $h=\exp \left(x_{1}\right) v$ with $v \in \mathbb{C}\left\{x_{2}\right\}$ i.e. $h=u x_{2}^{\ell}$ where $u \in \mathcal{O}$ is a unit. In particular, $h$ is weakly weighted-homogeneous (and weighted-homogeneous in fact).

Finally we prove 3$) \Rightarrow 1)$. Let $D=\alpha_{1} x_{1}\left(\partial / \partial x_{1}\right)+\alpha_{2} x_{2}\left(\partial / \partial x_{2}\right)$ be a semisimple operator such that $D h=d h$ with $d \in \mathbb{C}$. Without loss of generality, we may assume that $\alpha_{1}, \alpha_{2}, d \in \mathbb{Z}$ are integers.

Observe that, up to a change of coordinates, $h$ is a weighted-homogeneous polynomial. Indeed, it is clear if $\alpha_{1}$ and $\alpha_{2}$ are nonzero and have the same sign. Otherwise, if $\alpha_{1}=0$ then $h=v x_{2}^{a_{2}}$ with $v \in \mathbb{C}\left[\left[x_{1}\right]\right]$, i.e. $h=u x_{1}^{a_{1}} x_{2}^{a_{2}}$ with $u$ unit. Finally, when $\alpha_{1} \cdot \alpha_{2}<0$, the resolution of the Bezout identity $\alpha \cdot \gamma=d$ gives $h=x^{\gamma_{0}} v$ where $v \in \mathbb{C}\left[\left[x_{1}^{a_{1}} x_{2}^{a_{2}}\right]\right], \gamma_{0} \cdot \alpha=d$ and $a_{1} \alpha_{1}+a_{2} \alpha_{2}=0$ with $a_{1}, a_{2} \in \mathbb{N}^{*}, \operatorname{gcd}\left(a_{1}, a_{2}\right)=1$.

Hence, according to a theorem of Artin ([1]), there exists a convergent change of coordinates $\tilde{\phi}$ such that $h \circ \tilde{\phi}$ is a weighted-homogeneous polynomial.

3.2. Results on the suspension of a germ of plane curve. - An important fact in the proof of Theorem 1.6 is the explicit knowledge of the annihilators of $h^{s}$ and $\left(h+x_{3}^{N}\right)^{s}, N \in \mathbb{N}^{*}$, in the case of a germ $h$ of a plane curve (reduced or not).

LEMMA 3.5. - Let $h \in \mathbb{C}\left\{x_{1}, x_{2}\right\}$ be a nonzero germ with $h(0)=0$. Let $a_{1}$ (resp. $\left.a_{2}\right)$ denote the quotient of $h_{x_{1}}^{\prime}\left(\right.$ resp. $\left.h_{x_{2}}^{\prime}\right)$ by $\operatorname{gcd}\left(h_{x_{1}}^{\prime}, h_{x_{2}}^{\prime}\right)$.

(i) The ideal $\operatorname{Ann}_{\mathcal{D}} h^{s}$ is generated by $a_{2}\left(\partial / \partial x_{1}\right)-a_{1}\left(\partial / \partial x_{2}\right)$.

(ii) For all $N \in \mathbb{N}^{*}$, the ideal $\operatorname{Ann}_{\mathcal{D}}\left(h+x_{3}^{N}\right)^{s}$ is generated by

$$
\begin{array}{r}
N x_{3}^{N-1}\left(\partial / \partial x_{1}\right)-h_{x_{1}}^{\prime}\left(\partial / \partial x_{3}\right), \\
N x_{3}^{N-1}\left(\partial / \partial x_{2}\right)-h_{x_{2}}^{\prime}\left(\partial / \partial x_{3}\right), \\
a_{2}\left(\partial / \partial x_{1}\right)-a_{1}\left(\partial / \partial x_{2}\right) .
\end{array}
$$

Proof. - As the first point is easier than the second one, we will only prove (ii). Let us denote $I \subset \mathcal{D}$, the ideal generated by the given operators $S_{1}, S_{2}, S_{3}$. The inclusion $I \subset \operatorname{Ann}_{\mathcal{D}}\left(h+x_{3}^{N}\right)^{s}$ is obvious, so let us prove the reverse inclusion by induction on the order of operators.

TOME $132-2004-\mathrm{N}^{\mathrm{O}} 4$ 
Let $P \in \operatorname{Ann}_{\mathcal{D}}\left(h+x_{3}^{N}\right)^{s}$ be nonzero, of order $d$. As $d=0$ implies $P=0$, we will assume that $d \geq 1$. By division of $P$ by $S_{1}, S_{2}$, we get $R \in F_{d^{\prime}} \mathcal{D}, d^{\prime} \leq d$, with $P-R \in I$ and such that $\sigma(R) \in \mathcal{O}[\xi]$ may be written:

$$
\sigma(R)=v \xi_{3}^{d^{\prime}}+\sum_{i=1}^{d^{\prime}} \sum_{j=0}^{N-2} \Upsilon_{i}\left(\xi_{1}, \xi_{2}\right) x_{3}^{j} \xi_{3}^{d^{\prime}-i}
$$

with $v \in \mathcal{O}$ and $\Upsilon_{i} \in \mathbb{C}\left\{x_{1}, x_{2}\right\}\left[\xi_{1}, \xi_{2}\right]$ are zero or homogeneous in $\left(\xi_{1}, \xi_{2}\right)$ of degree $i$. Remark that $R$ annihilates $\left(h+x_{3}^{N}\right)^{s}$. If $d^{\prime}<d$, then $R$ belongs to $I$ by induction, and so does $P$. Otherwise, by an easy computation, we get $\sigma(R)\left(h_{x_{1}}^{\prime}, h_{x_{2}}^{\prime}, N x_{3}^{N-1}\right)=0$. Thus, studying the coefficient of the powers of $x_{3}$, we have $v=0$ and the homogeneous polynomials $\Upsilon_{i}\left(\xi_{1}, \xi_{2}\right)$ are zero on $\left(h_{x_{1}}^{\prime}, h_{x_{2}}^{\prime}\right)$, i.e. on the regular sequence $\left(a_{1}, a_{2}\right)$. Hence, the polynomials $\Upsilon_{i}\left(\xi_{1}, \xi_{2}\right)$ are multiples of $\sigma\left(S_{3}\right)$ and there exists a homogeneous polynomial $A \in \mathcal{O}[\xi]$ such that $\sigma(R)=A \sigma\left(S_{3}\right)$. If $\widetilde{A} \in F_{d-1} \mathcal{D}$ is such that $\sigma(\widetilde{A})=A$, then $R-\widetilde{A} S_{3}$ belongs to $F_{d-1} \mathcal{D}$ and annihilates $\left(h+x_{3}^{N}\right)^{s}$. By induction, it belongs to $I$, and so do $R$ and $P$.

Now we give a result on the Bernstein polynomial of the suspension of a weighted-homogeneous plane curve.

LEMMA 3.6. - Let $h \in \mathbb{C}\left\{x_{1}, x_{2}\right\}$ be a weighted-homogeneous germ and $N$ an integer greater than or equal to 2 . Let us denote by $\tilde{b}(s)$ (resp. $\left.\tilde{b}_{N}(s)\right)$ the reduced Bernstein polynomial of $h\left(\right.$ resp. $\left.h+x_{3}^{N}\right)$. Then $\tilde{b}_{N}(s)$ divides $\prod_{i=1}^{N-1} \tilde{b}(s+i / N)$, and $\tilde{b}_{N}(s)$ is a multiple of the polynomials $\tilde{b}(s+i / N), 1 \leq i \leq N-1$. In particular, $\prod_{i=1}^{N-1} \tilde{b}(s+i / N)$ and $\tilde{b}_{N}(s)$ have the same roots.

Proof. - Let $\chi \in \widetilde{\mathcal{D}}=\mathbb{C}\left\{x_{1}, x_{2}\right\}\left\langle\partial / \partial x_{1}, \partial / \partial x_{2}\right\rangle$, be the Euler-vector field such that $\chi \cdot h^{s}=s h^{s}$. Using the results recalled in $\S 2$ and the previous lemma, it is easy to check that the functional equations defining $\tilde{b}(s)$ and $\tilde{b}_{N}(s)$ may be written:

and

$$
c(\chi) \in \widetilde{\mathcal{D}}\left(h_{x_{1}}^{\prime}, h_{x_{2}}^{\prime}, a_{2} \frac{\partial}{\partial x_{1}}-a_{1} \frac{\partial}{\partial x_{2}}\right)=\widetilde{I}
$$

$$
c\left(\chi+\frac{x_{3}}{N} \frac{\partial}{\partial x_{3}}\right) \in \mathcal{D}\left(h_{x_{1}}^{\prime}, h_{x_{2}}^{\prime}, x_{3}^{N-1}, a_{2} \frac{\partial}{\partial x_{1}}-a_{1} \frac{\partial}{\partial x_{2}}\right)=I .
$$

In particular, $\tilde{b}(\chi) \in I$. Hence, in order to get the first point, we just have to prove that $P=\prod_{i=1}^{N-1} \tilde{b}\left(\chi+\left(x_{3} / N\right)\left(\partial / \partial x_{3}\right)+i / N\right)$ belongs to $I$. Observe that $P$ may be written:

$$
\prod_{i=1}^{N-1} \tilde{b}\left(\chi+\frac{\partial}{\partial x_{3}} \frac{x_{3}}{N}+\frac{i-1}{N}\right)=\left[\prod_{i=2}^{N-1} \tilde{b}\left(\chi+\frac{\partial}{\partial x_{3}} \frac{x_{3}}{N}+\frac{i-1}{N}\right)\right] \sum_{k \geq 0} \frac{\tilde{b}^{(k)}(\chi)}{k !}\left(\frac{\partial}{\partial x_{3}} \frac{x_{3}}{N}\right)^{k}
$$

BULletin DE LA SOCiÉtÉ MATHÉmAtique DE FRANCE 
using the Taylor formula. So, up to a multiple of $\tilde{b}(\chi), P$ may be rewritten:

$$
\frac{\partial}{\partial x_{3}}\left[\sum_{k \geq 1} \frac{\tilde{b}^{(k)}(\chi)}{k !}\left(\frac{\partial}{\partial x_{3}} \frac{x_{3}}{N}-\frac{1}{N}\right)^{k-1}\right] \cdot\left[\prod_{i=1}^{N-2} \tilde{b}\left(\chi+\frac{\partial}{\partial x_{3}} \frac{x_{3}}{N}+\frac{i-1}{N}\right)\right] \frac{x_{3}}{N} .
$$

Iterating this process, we check that $P$ belongs to $\mathcal{D}\left(\tilde{b}(\chi), x_{3}^{N-1}\right) \subset I$.

Now we prove the last part. Multiplying identity (3) by $x_{3}^{i-1}$ on the left, $1 \leq i \leq N-1$, we get:

$$
\tilde{b}_{N}\left(\chi+\frac{\partial}{\partial x_{3}} \frac{x_{3}}{N}-\frac{i}{N}\right) x_{3}^{i-1} \in I .
$$

Thus, using Taylor's formula, we have $\tilde{b}_{N}(\chi-i / N) x_{3}^{i-1} \in I+\mathcal{D} x_{3}^{i}$. Observe that each element of $I+\mathcal{D} x_{3}^{i}$ may be written in a unique way:

$$
P x_{3}^{i}+\sum_{j=0}^{J} \sum_{k=0}^{i-1}\left(\frac{\partial}{\partial x_{3}}\right)^{j} P_{j, k} x_{3}^{k},
$$

where $P \in \mathcal{D}, P_{k, j} \in \widetilde{I}, j \in \mathbb{N}$. Hence the operator $\tilde{b}_{N}(\chi-i / N)$ belongs to $\widetilde{I}$, i.e. the polynomials $\tilde{b}(s+i / N), 1 \leq i \leq N-1$, divide $\tilde{b}_{N}(s)$.

3.3. Proof of Theorem 1.6. - Again we only prove (ii). Without loss of generality, we will assume that $h$ is singular (since the assertion is clear when $h+x_{3}^{N}$ is smooth).

We recall that roots of the Bernstein polynomial of a holomorphic function on $\mathbb{C}^{n}$ are included in $]-n, 0[$ (see [15], [20]). In particular, the condition (b) means that the smallest integral root of the Bernstein polynomial of $h+x_{3}^{N}$ is strictly greater than $-\ell-1$ (see Lemma 3.6). Moreover, as $\operatorname{Ann}_{\mathcal{D}}\left(h+x_{3}^{N}\right)^{s}$ is generated by operators of order 1 (Lemma 3.5), the condition on $1 /\left(h+x_{3}^{N}\right)^{\ell}$ is true when $h$ is weighted-homogeneous (see the introduction).

Conversely, let us assume that $\operatorname{Ann}_{\mathcal{D}} 1 /\left(h+x_{3}^{N}\right)^{\ell}$ is generated by the operators $Q_{1}, \ldots, Q_{w} \in F_{1} \mathcal{D}$. From Proposition 1.3, we have to prove that $h$ is weighted-homogeneous. Let $q_{i}$ be the germ $Q_{i}(1) \in \mathcal{O}$ and $Q_{i}^{\prime} \in \mathcal{D}$ the vector field $Q_{i}-q_{i}$. Then we have:

$$
-\ell Q_{i}^{\prime}\left(h+x_{3}^{N}\right)+\left(h+x_{3}^{N}\right) q_{i}=0, \quad 1 \leq i \leq w .
$$

On the other hand, from Lemma 3.3 of [18], there exists an operator $R$ in $\operatorname{Ann}_{\mathcal{D}}\left(h+x_{3}^{N}\right)^{s}$ such that $R=1+\sum_{i=1}^{w} A_{i} q_{i}$, with $A_{i} \in \mathcal{D}$. It comes from the division by $Q_{1}, \ldots, Q_{w}$ of a good operator of $\operatorname{Ann}_{\mathcal{D}[s]}\left(h+x_{3}^{N}\right)^{s}$ (see [11, Thm 6.3]), i.e. of the form $s^{N}+\sum_{i=0}^{N-1} s^{i} P_{i}$ with $P_{i} \in F_{N-i} \mathcal{D}, 0 \leq i \leq N-1$. Considering the constant coefficient of $R$ in the writting with coefficients on the right, we get:

$$
1 \in\left(q_{1}, \ldots, q_{w}, x_{3}^{N-1}, a_{1}, a_{2}, \frac{\partial\left(a_{2}\right)}{\partial x_{1}}-\frac{\partial\left(a_{1}\right)}{\partial x_{2}}\right) \mathcal{O}
$$

TOME $132-2004-\mathrm{N}^{\mathrm{O}} 4$ 
with the help of the determination of $\operatorname{Ann}_{\mathcal{D}}\left(h+x_{3}^{N}\right)^{s}$ (Lemma 3.5). Thus, at most one of the generators of this ideal is a unit. If $q_{i}$ is a unit, from (4), the germ $h+x_{3}^{N}$ is Euler-homogeneous and so does $h$. We conclude with Proposition 3.4 .

If $a_{1}$ or $a_{2}$ is a unit, the operator $D=a_{2}\left(\partial / \partial x_{1}\right)-a_{1}\left(\partial / \partial x_{2}\right) \in \operatorname{Ann}_{\mathcal{D}} h$ is regular. So, up to a change of coordinates, $h$ belongs to $\mathbb{C}\left\{x_{2}\right\}$, and so it is weighted-homogeneous.

Finally, let us suppose that $D$ is singular with $\partial\left(a_{2}\right) / \partial x_{1}-\partial\left(a_{1}\right) / \partial x_{2}$ a unit. Thus, the formal operator $D$ is not nilpotent. From Proposition 3.1, there exists a formal change of coordinates such that $D=D_{S}+D_{N}$ with $D_{S}=\alpha_{1} \tilde{x}_{1}\left(\partial / \partial \tilde{x}_{1}\right)+\alpha_{2} \tilde{x}_{2}\left(\partial / \partial \tilde{x}_{2}\right) \neq 0$. Thus, as $D h=0, h$ is weakly weightedhomogeneous of type $\left(0, \alpha_{1}, \alpha_{2}\right)$ in the coordinates $\left(\tilde{x}_{1}, \tilde{x}_{2}\right)$ (Proposition 3.2). In particular, $h$ is weighted-homogeneous (Proposition 3.4).

\section{A companion piece to Theorem 1.2}

In this part, we adapt the proof of Theorem 1.2 in order to characterize the germs $h \in \mathcal{O}$ such that $\operatorname{Ann}_{\mathcal{D}} 1 / h^{\ell}$ is generated by operators of order 1 in some cases where $h$ has non isolated singularities. First we prove Theorem 1.7, where we take good assumptions on $\operatorname{gr}^{F} \operatorname{Ann}_{\mathcal{D}} 1 / h^{\ell}$ in order to have a division with control of the orders (see $\S 3.3$ ). Then we get a full characterization in the case of Koszul-free germs (Corollary 1.8).

4.1. Proof of Theorem 1.7. - According to the introduction, it is enough to check the following result.

Proposition 4.1. - Let $h \in \mathcal{O}$ be a nonzero germ with $h(0)=0$, and let $\ell \in \mathbb{N}^{*}$ be a nonnegative integer. Let us suppose that $\mathrm{Ann}_{\mathcal{D}} 1 / h^{\ell}$ is generated by some operators $Q_{1}, \ldots, Q_{w} \in \mathcal{D}$ of order 1 such that:

$$
\operatorname{gr}^{F} \operatorname{Ann}_{\mathcal{D}} 1 / h^{\ell}=\left(\sigma\left(Q_{1}\right), \ldots, \sigma\left(Q_{w}\right)\right) \operatorname{gr}^{F} \mathcal{D} .
$$

Then the following conditions are verified:

(a) the germ $h$ belongs to the ideal of its partial derivatives,

(b) the smallest integral root of the Bernstein polynomial of $h$ is strictly greater than $-\ell-1$,

(c) the ideal $\operatorname{Ann}_{\mathcal{D}} h^{s}$ is generated by operators of order 1.

Moreover, $\operatorname{Ann}_{\mathcal{D}} h^{s}$ is also generated by $Q_{j}(1) Q_{i}-Q_{i}(1) Q_{j}, 1 \leq i \leq w, i \neq j$, where $j$ is such that $Q_{j}(1)$ is a unit.

Proof. - First, it is easy to check that the assumption on $\operatorname{gr}^{F} \mathrm{Ann}_{\mathcal{D}} 1 / h^{\ell}$ means: every $P \in \operatorname{Ann}_{\mathcal{D}} 1 / h^{\ell}$ of order $d$ may be written $P=\sum_{i=1}^{w} A_{i} Q_{i}$ where $A_{i} Q_{i}$ belongs to $F_{d} \mathcal{D}$. 
The first part of the proof uses the main idea of Lemma 3 of [18]. For $1 \leq i \leq w$, let us denote by $q_{i}$ the germ $Q_{i}(1) \in \mathcal{O}$ and by $Q_{i}^{\prime} \in \mathcal{D}$ the vector field $Q_{i}-q_{i}$. Thus we have:

$$
\ell Q_{i}^{\prime}(h)-q_{i} h=0, \quad 1 \leq i \leq w .
$$

Let $P_{0}(s) \in \mathcal{D}[s]$ be a good operator in $s$ of order $N$ such that $P_{0}(s) \cdot h^{s}=0$ (see [11]). By division, it may be written:

$$
P_{0}(s)=(s+\ell) R_{0}(s)+P_{0}(-\ell),
$$

where $R_{0}(s)$ is a good operator of order $N-1$ and $P_{0}(-\ell)$ annihilates $1 / h^{\ell}$. So $P_{0}(-\ell)$ is equal to $\sum_{i=1}^{\ell} A_{i} Q_{i}$ with $A_{i} \in F_{N-1} \mathcal{D}, 1 \leq i \leq w$. Hence:

$$
(s+\ell) R_{0}(s) h^{s}+\frac{(s+\ell)}{\ell} \sum_{i=1}^{w} A_{i} q_{i} h^{s}=0 .
$$

So $P_{1}(s)=R_{0}(s)+(1 / \ell) \sum_{i=1}^{w} A_{i} q_{i}$ is a good operator in $s$ of order $N-1$ such that $P_{1}(s) \cdot h^{s}=0$. Iterating this process, we may assume $N=1$. So there exists $a_{i} \in \mathcal{O}$ such that $1+\sum_{i=1}^{w} a_{i} q_{i}=0$; in particular, at most one of the $q_{i}$ is a unit. From (5), we deduce that $h$ belongs to the ideal of its partial derivatives. Without loss of generality, we will assume that $q_{1}=1$.

Now, we will prove the assertion about $\operatorname{Ann}_{\mathcal{D}} h^{s}$. As the given operators clearly annihilate $h^{s}$, it is enough to prove that every $P \in \operatorname{Ann}_{\mathcal{D}} h^{s}$ belongs to the ideal generated by $Q_{i}-q_{i} Q_{1}, 2 \leq i \leq w$. We do it by induction on the order $d \in \mathbb{N}$ of $P$.

If $d=0$, then $P=0$ and the assertion is true. Otherwise, as $P$ annihilates $1 / g^{\ell}$, we have:

$$
P=\sum_{i=1}^{w} A_{i}^{(0)} Q_{i}=\sum_{i=2}^{w} A_{i}^{(0)}\left(Q_{i}-q_{i} Q_{1}\right)+\underbrace{\left(A_{1}^{(0)}+\sum_{i=2}^{w} A_{i}^{(0)} q_{i}\right.}_{P^{\prime}}) Q_{1}
$$

with $P^{\prime}, A_{1}^{(0)}, \ldots, A_{w}^{(0)} \in F_{d-1} \mathcal{D}$. Remark that $P^{\prime} \in \operatorname{Ann}_{\mathcal{D}} h^{s}\left(\right.$ since $\left.Q_{1}\left(h^{s}\right)=s h^{s}\right)$. Iterating this process, we get:

$$
P=\underbrace{\sum_{i=2}^{w} \sum_{k=0}^{d-1} A_{i}^{(k)}\left(Q_{i}-q_{i} Q_{1}\right) Q_{1}^{k}}_{\widetilde{P}}+p Q_{1}^{d}
$$

with $A_{i}^{(k)} \in F_{d-k-1} \mathcal{D}$ and $p \in \mathcal{O}$. But $p$ must be zero because $P, \widetilde{P} \in \operatorname{Ann}_{\mathcal{D}} h^{s}$. Thus:

$$
P=\sum_{i=2}^{w} \sum_{k=0}^{d-1} A_{i}^{(k)} Q_{1}^{k}\left(Q_{i}-q_{i} Q_{1}\right)+\underbrace{\sum_{i=2}^{w} \sum_{k=0}^{d-1} A_{i}^{(k)}\left[Q_{i}-q_{i} Q_{1}, Q_{1}^{k}\right]}_{R}
$$

with $R \in \operatorname{Ann}_{\mathcal{D}} h^{s} \cap F_{d-1} \mathcal{D}$. We conclude by induction.

TOME $132-2004-\mathrm{N}^{\mathrm{O}} 4$ 
REMARK 4.2. - In fact, we have proved that $\operatorname{gr}^{F} \mathrm{Ann}_{\mathcal{D}} h^{s}$ is generated by the principal symbols of the given operators.

4.2. The case of Koszul-free germs. - First we prove that the Koszulfree germs verify the assumption of Theorem 1.7.

Lemma 4.3. - Let $h \in \mathcal{O}$ be a Koszul-free germ. Then there exists $Q_{1}, \ldots, Q_{n} \in F_{1} \mathcal{D}$ generating $\operatorname{Ann}_{\mathcal{D}} 1 / h \cap F_{1} \mathcal{D}$, such that:

$$
\operatorname{gr}^{F} \mathcal{D}\left(Q_{1}, \ldots, Q_{n}\right)=\left(\sigma\left(Q_{1}\right), \ldots, \sigma\left(Q_{n}\right)\right) \operatorname{gr}^{F} \mathcal{D}
$$

Proof. - Let $\left\{\delta_{1}, \ldots, \delta_{n}\right\}$ be a basis of $\operatorname{Der}(\log h)$ such that $\left\{\sigma\left(\delta_{1}\right), \ldots, \sigma\left(\delta_{n}\right)\right\}$ is a $\operatorname{gr}^{F} \mathcal{D}$-regular sequence. For $1 \leq i \leq n$, let $a_{i} \in \mathcal{O}$ be the germ defined by $\delta_{i}(h)=a_{i} h$ and let $Q_{i} \in \operatorname{Ann}_{\mathcal{D}} 1 / h \cap F_{1} \mathcal{D}$ be the operator $\delta_{i}+a_{i}$. Using that $\delta_{1}, \ldots, \delta_{n}$ generate $\operatorname{Der}(\log h)$, it is easy to check that $Q_{1}, \ldots, Q_{n}$ generate $\operatorname{Ann}_{\mathcal{D}} 1 / h \cap F_{1} \mathcal{D}$. On the other hand, $\operatorname{gr}^{F} \mathcal{D}\left(Q_{1}, \ldots, Q_{n}\right)$ is generated by $\sigma\left(Q_{i}\right)=\sigma\left(\delta_{i}\right), 1 \leq i \leq n$, because this family defines a regular sequence (see [3, Prop. 4.1.2] and [17, Lemma 2]).

Let us recall some facts about logarithmic operators (see $[3, \S 1.2]$ ). Given a nonzero germ $h \in \mathcal{O}$ such that $h(0)=0$, a differential operator $P \in \mathcal{D}$ is logarithmic with respect to $h$ if $P \cdot h^{k} \mathcal{O} \subset h^{k} \mathcal{O}$ for all $k \in \mathbb{Z}$. Let us denote by $\mathcal{V}_{0}^{h}(\mathcal{D}) \subset \mathcal{D}$ the subring of differential logarithmic operators. When $h$ is free, $\mathcal{V}_{0}^{h}(\mathcal{D})$ is a coherent sheaf of rings ([3, Cor. 2.1.7]).

Now we characterize the condition (c) for Euler-homogeneous free germs.

Proposition 4.4. - Let $h \in \mathcal{O}$ be an Euler-homogeneous free germ, and let $\left\{\delta_{1}, \ldots, \delta_{n}\right\}$ be a basis of $\operatorname{Der}(\log h)$ such that $\delta_{1}(h)=h$ and $\delta_{i}(h)=0$ for $2 \leq i \leq n$. We denote by $I \subset \mathcal{D}$ the ideal generated by $\delta_{2}, \ldots, \delta_{n}$. The following conditions are equivalent:

1) the ideal $\mathrm{Ann}_{\mathcal{D}} h^{s}$ is generated by operators of order 1 ,

2) the ideal $\operatorname{Ann}_{\mathcal{D}} h^{s}$ is generated by logarithmic differential operators,

3) the ideal $\mathrm{Ann}_{\mathcal{D}} h^{s}$ coincides with the ideal $I$.

Moreover, if $\operatorname{gr}^{F} I$ is generated by $\sigma\left(\delta_{2}\right), \ldots, \sigma\left(\delta_{n}\right)$, then these conditions are equivalent to:

4) the sequence $\left(h, \sigma\left(\delta_{2}\right), \ldots, \sigma\left(\delta_{n}\right)\right)$ is $\operatorname{gr}^{F} \mathcal{D}$-regular.

Proof. - The implication 2) $\Rightarrow 3$ ) is a consequence of Lemma 4.6. As 3) $\Rightarrow 1$ ) is obvious, let us remark that 1) implies 2). Indeed, if $P \in F_{1} \mathcal{D}$ annihilates $h^{s}$, then, for all $a \in \mathcal{O}, k \in \mathbb{Z}$, we have $P\left(a h^{k}\right)=P(a) h^{k}$.

Now, we prove 4$) \Rightarrow 3$ ). Observe that condition 4) implies $\operatorname{gr}^{F} I=$ $\left(\sigma\left(\delta_{2}\right), \ldots, \sigma\left(\delta_{n}\right)\right) \operatorname{gr}^{F} \mathcal{D}$ (see $[17$, Lemma 2$]$ ). Let $P \in \mathcal{D}$ be an operator annihilating $h^{s}$. So there exists $N \in \mathbb{N}$ such that $h^{N} P \in \mathcal{V}_{0}^{h}(\mathcal{D})$, hence $h^{N} P \in I$ by Lemma 4.6. If $N=0$, the assertion is obvious. Otherwise, from our 
assumption, the endomorphism of $\operatorname{gr}^{F} \mathcal{D} / \operatorname{gr}^{F} I$ induced by $h$ is one to one. We deduce that $h^{N-1} P \in I$ with the help of the following result:

Lemma 4.5 ([16, Lemme 4.3.2.6]). - Let $U \in \mathcal{D}$ and let $I \subset \mathcal{D}$ be an ideal, such that the endomorphism of $\operatorname{gr}^{F} \mathcal{D} / \operatorname{gr}^{F} I$ induced by the multiplication by $\sigma(U)$ in $\operatorname{gr}^{F} \mathcal{D}$ is one-to-one. If $R \in \mathcal{D}$ is such that $U R \in I$, then $R \in I$.

Therefore, $P \in I$ by induction on $N$.

Finally, we prove the implication 3$) \Rightarrow 4$ ) under the following assumption:

$$
\operatorname{gr}^{F} I=\left(\sigma\left(\delta_{2}\right), \ldots, \sigma\left(\delta_{n}\right)\right) \operatorname{gr}^{F} \mathcal{D} .
$$

Let us recall that the characteristic variety of $\mathcal{D} h^{s}$ is the relative conormal space associated with $h$, which is the subspace $W_{h} \subset T^{*} \mathbb{C}^{n}$ defined as the closure in $T^{*} \mathbb{C}^{n}$ of $\{(x, \lambda \mathrm{d} h(x)) ; \lambda \in \mathbb{C}\}([11])$. In particular, $W_{h}$ is irreducible of pure dimension $n+1$. From the principal ideal theorem, $W_{0}(h)=W_{h} \cap\{h=0\}$ has pure dimension $n$. So, if $I=\operatorname{Ann}_{\mathcal{D}} h^{s}$ then $\operatorname{gr}^{F} I+h \operatorname{gr}^{F} \mathcal{D}$ defines $W_{0}(h)$ and $\left(h, \sigma\left(\delta_{2}\right), \ldots, \sigma\left(\delta_{n}\right)\right)$ is a regular sequence.

LEMMA 4.6. - Let $h \in \mathcal{O}$ be an Euler-homogeneous free germ, and let $\left\{\delta_{1}, \ldots, \delta_{n}\right\}$ be a basis of $\operatorname{Der}(\log h)$ such that $\delta_{1}(h)=h$ and $\delta_{i}(h)=0$ for $2 \leq i \leq n$. Then the ideal $\operatorname{Ann}_{\mathcal{V}_{0}^{h}(\mathcal{D})} h^{s}$ coincides with $\mathcal{V}_{0}^{h}(\mathcal{D})\left(\delta_{2}, \ldots, \delta_{n}\right)$.

Proof. - From the structure theorem of logarithmic operators (see [3, Thm 2.1.4]), each logarithmic operator of order $d$ may be written in a unique way $\sum_{|\gamma| \leq d} a_{\gamma} \delta_{1}^{\gamma_{1}} \cdots \delta_{n}^{\gamma_{n}}, a_{\gamma} \in \mathcal{O}$. Thus the assertion is a consequence of the identities $\delta_{1} \cdot h^{s}=s h^{s}$ and $\delta_{i} \cdot h^{s}=0,2 \leq i \leq n$.

REMARK 4.7. - We do not know if condition (b) of Corollary 1.8 is - or not always true when $h$ is Koszul-free, or Koszul-free and verifying (a) \& (c).

\section{The case of generic arrangements of hyperplanes}

The purpose of this part is to prove that $\operatorname{Ann}_{\mathcal{D}} 1 / h$ is generated by operators of order 1 when $h \in \mathbb{C}\left[x_{1}, \ldots, x_{n}\right]$ defines a particular type of central hyperplane arrangement $\mathcal{A} \subset \mathbb{C}^{n}$ (Theorem 5.2). As the case $n=2$ is a consequence of Theorem 1.6, we will assume that $n \geq 3$.

Recall that a (central) hyperplane arrangement defined by $\prod_{i=1}^{p} \ell_{i}=0$, with $p \geq 2$ and $\ell_{i} \in\left(\mathbb{C}^{n}\right)^{*}$, is:

- generic if $p \geq n$ and if, for all $1 \leq i_{1}<\cdots<i_{n} \leq p,\left(\ell_{i_{1}}, \ldots, \ell_{i_{n}}\right)$ defines the origin;

- hyperbolic if $\ell_{i} \in \mathbb{C} \ell_{1}+\mathbb{C} \ell_{2}$ for $3 \leq i \leq p$.

Notation 5.1. - Let $f=\left(f_{1}, \ldots, f_{r}\right): \mathbb{C}^{n} \rightarrow \mathbb{C}^{r}, 1 \leq r<n$, be an analytic morphism. For every multi-index $K=\left(k_{1}, \ldots, k_{r+1}\right) \in \mathbb{N}^{r+1}$ where

TOME $132-2004-\mathrm{N}^{\mathrm{O}} 4$ 
$1 \leq k_{1}, \ldots, k_{r+1} \leq n$ and $k_{i} \neq k_{j}$ for $i \neq j$, let $\Delta_{K}^{f_{1}, \ldots, f_{r}} \in \mathcal{D}$ denote the vector field:

$$
\sum_{i=1}^{r+1}(-1)^{i} m_{K(i)}(f) \frac{\partial}{\partial x_{k_{i}}}=\sum_{i=1}^{r+1}(-1)^{i} \frac{\partial}{\partial x_{k_{i}}} m_{K(i)}(f),
$$

where $K(i)=\left(k_{1}, \ldots, \check{k}_{i}, \ldots, k_{r+1}\right) \in \mathbb{N}^{r}$ and $m_{K(i)}(f)$ is the determinant of the $r \times r$ matrix obtained from the jacobian matrix of $f$ by deleting the $k$-th columns with $k \notin\left\{k_{1}, \ldots, \check{k_{i}}, \ldots, k_{r+1}\right\}$.

In the particular case $r=n-1$, the only vector field is denoted by $\Delta^{f_{1}, \ldots, f_{r}}$.

THEOREM 5.2. - Let $\mathcal{A} \subset \mathbb{C}^{n}, n \geq 3$, be a central generic arrangement of hyperplanes, defined by $h=\prod_{i=1}^{p} \ell_{i}, p \geq n$. Let $\mathcal{A}^{\prime} \subset \mathbb{C}^{n}$ be a hyperbolic arrangement defined by: $h^{\prime}=\prod_{i=1}^{q} \ell_{i}^{\prime}, q \geq 2$, and such that $\ell_{i}^{\prime} h=0$ defines $a$ generic arrangement for all $1 \leq i \leq q$.

Then $\operatorname{Ann}_{\mathcal{D}} 1 / h^{\prime} h$ is generated by $\sum_{i=1}^{n} x_{i}\left(\partial / \partial x_{i}\right)+p+q$ and by:

$$
\Delta^{h^{\prime}, \ell_{i_{1}}, \ldots, \ell_{i_{n-2}}} \cdot \prod_{i \neq i_{1}, \ldots, i_{n-2}} \ell_{i} \quad \& \quad \Delta^{\ell_{1}^{\prime}, \ell_{2}^{\prime}, \ell_{i_{1}}, \ldots, \ell_{i_{n-3}}} \cdot \prod_{\ell_{i} \notin \mathbb{C}\left(\ell_{1}^{\prime}, \ell_{2}^{\prime}, \ell_{i_{1}}, \ldots, \ell_{i_{n-3}}\right)} \ell_{i}
$$

for all family of distinct indexes $1 \leq i_{1}<\cdots<i_{n-3} \leq p, 1 \leq i_{n-2} \leq p$, such that $\left(\ell_{1}^{\prime}, \ell_{2}^{\prime}, \ell_{i_{1}}, \ldots, \ell_{i_{n-2}}\right)$ defines the origin.

As an easy consequence, we have the following result.

Corollary 5.3. - Let $\mathcal{A} \subset \mathbb{C}^{n}, n \geq 3$, be a central generic arrangement of hyperplanes, defined by $h=\prod_{i=1}^{p} \ell_{i}, p \geq n$. Then the ideal $\mathrm{Ann}_{\mathcal{D}} 1 / h$ is generated by $\sum_{i=1}^{n} x_{i} \frac{\partial}{\partial x_{i}}+p$ and by the operators:

$$
\Delta^{\ell_{i_{1}}, \ldots, \ell_{i_{n-1}}} \cdot \prod_{i \neq i_{1}, \ldots, i_{n-1}} \ell_{i}
$$

for all $1=i_{1}<\cdots<i_{n-1} \leq p$.

The proof of Theorem 5.2 needs the following technical computation.

Proposition 5.4. - Let $\ell_{1}, \ldots, \ell_{p} \in\left(\mathbb{C}^{n}\right)^{*}, 3 \leq n \leq p$, be linear forms which define a generic arrangement and $h \in \mathbb{C}\left[x_{1}, \ldots, x_{n}\right]$ be the product $\prod_{i=1}^{p} \ell_{i}$. Let $g \in \mathbb{C}\left\{x_{1}, x_{2}\right\}$ be a reduced germ such that $\left(g, \ell_{i_{1}}, \ldots, \ell_{i_{n-1}}\right)$ defines the origin for all $1 \leq i_{1}<\cdots<i_{n-1} \leq p$. Then the ideal $\operatorname{Ann}_{\mathcal{D}}(1 / h) g^{s}$ is generated by the operators:

$$
\Delta^{g, \ell_{i_{1}}, \ldots, \ell_{i_{n-2}}} \cdot \prod_{i \neq i_{1}, \ldots, i_{n-2}} \ell_{i} \text { and } \Delta^{x_{1}, x_{2}, \ell_{i_{1}}, \ldots, \ell_{i_{n-3}}} \cdot \prod_{\ell_{i} \notin \mathbb{C}\left(x_{1}, x_{2}, \ell_{i_{1}}, \ldots, \ell_{i_{n-3}}\right)} \ell_{i}
$$

for all sequence of distinct indexes $i_{1}, \ldots, i_{n-2}$ such that $\left(x_{1}, x_{2}, \ell_{i_{1}}, \ldots, \ell_{i_{n-2}}\right)$ defines the origin. 
REMARK 5.5. - If $\ell_{i_{1}}, \ldots, \ell_{i_{k}}, 1 \leq k \leq n-3$, are some linear factors of $h$ such that the family $\left(x_{1}, x_{2}, \ell_{i_{1}}, \ldots, \ell_{i_{k}}\right)$ is free, then there exists at most one form $\ell_{i}, i \neq i_{1}, \ldots, i_{k}$, such that $\ell_{i}$ belongs to the space $\mathbb{C}\left(x_{1}, x_{2}, \ell_{i_{1}}, \ldots, \ell_{i_{k}}\right)=$ $\mathbb{C} x_{1}+\mathbb{C} x_{2}+\mathbb{C} \ell_{i_{1}}+\cdots+\mathbb{C} \ell_{i_{k}} \subset\left(\mathbb{C}^{n}\right)^{*}$. Indeed, if $\ell_{i_{k+1}}$ and $\ell_{i_{k+2}}$ are two such forms, then $\mathbb{C}\left(x_{1}, x_{2}, \ell_{i_{1}}, \ldots, \ell_{i_{k}}\right)=\mathbb{C}\left(\ell_{i_{1}}, \ldots, \ell_{i_{k+2}}\right)$ since $\mathcal{A}$ is generic. And this is not possible because $\left(g, \ell_{i_{1}}, \ldots, \ell_{i_{k+2}}\right)$ is $\mathcal{O}$-regular (and $g \in \mathbb{C}\left\{x_{1}, x_{2}\right\}$ ). Conversely, if there exists a form $\ell_{i}, i \neq i_{1}, \ldots, i_{k}$ (with $k \leq n-2$ ), such that $\ell_{i} \in \mathbb{C}\left(x_{1}, x_{2}, \ell_{i_{1}}, \ldots, \ell_{i_{k}}\right)$, we prove by a similar argument that the family $\left(x_{1}, x_{2}, \ell_{i_{1}}, \ldots, \ell_{i_{k}}\right)$ must be free.

Proof of Proposition 5.4. - Let us denote $I \subset \mathcal{D}$ the left ideal generated by the given operators and $\mathcal{I} \subset \mathcal{O}\left[\xi_{1}, \ldots, \xi_{n}\right]$ the ideal generated by their principal symbols. First we prove the following fact.

AsSERTION 1. - The ideal I contains the operators of the form:

$$
v \cdot \prod_{i \neq i_{1}, \ldots, i_{k}} \ell_{i}
$$

with $0 \leq k \leq n-2,1 \leq i_{1}<\cdots<i_{k} \leq p$, and $v \in \mathcal{D}$ is a vector field annihilating $g, \ell_{i_{1}}, \ldots, \ell_{i_{k}}$.

Proof. — Two cases are possible.

First, let us suppose that the family $\left(x_{1}, x_{2}, \ell_{i_{1}}, \ldots, \ell_{i_{k}}\right)$ is free. If $k<n-2$, we can find some other linear forms $\ell_{i_{k+1}}, \ldots, \ell_{i_{n-2}}$ of the arrangement such that $\left(x_{1}, x_{2}, \ell_{i_{1}}, \ldots, \ell_{i_{n-2}}\right)$ defines the origin (Remark 5.5). Then we have:

$$
v=v_{1}+\underbrace{\sum_{j=k+1}^{n-2} \frac{v\left(\ell_{i_{j}}\right)}{\Delta^{x_{1}, x_{2}, \ell_{i_{1}}, \ldots, \check{\ell}_{i_{j}}, \ldots, \ell_{i_{n-2}}\left(\ell_{i_{j}}\right)}} \Delta^{x_{1}, x_{2}, \ell_{i_{1}}, \ldots, \check{\ell}_{i_{j}}, \ldots, \ell_{i_{n-2}}}}_{v_{2}}
$$

where $v_{1}$ annihilates $g, \ell_{i_{1}}, \ldots, \ell_{i_{n-2}} ;$ thus $v_{1} \in \mathcal{O} \Delta^{g, \ell_{i_{1}}, \ldots, \ell_{i_{n-2}}}$ by an easy computation in the coordinates $\left(x_{1}, x_{2}, \ell_{i_{1}}, \ldots, \ell_{i_{n-2}}\right)$. In particular, $v_{1} \prod_{i \neq i_{1}, \ldots, i_{k}} \ell_{i}$ belongs to:

$$
\mathcal{D}\left(\prod_{i=k+1}^{n-2} \ell_{i}\right) \Delta^{g, \ell_{i_{1}}, \ldots, \ell_{i_{n-2}}}\left(\prod_{i \neq i_{1}, \ldots, i_{n-2}} \ell_{i}\right) \subset I .
$$

By similar computations, we check that $v_{2} \prod_{i \neq i_{1}, \ldots, i_{k}} \ell_{i}$ belongs to $I$; thus so does $v \prod_{i \neq i_{1}, \ldots, i_{k}} \ell_{i}$.

Now assume that the family $\left(x_{1}, x_{2}, \ell_{i_{1}}, \ldots, \ell_{i_{k}}\right)$ is not free. As the sequence $\left(g, \ell_{i_{1}}, \ldots, \ell_{i_{k}}\right)$ is regular, we can not have $x_{1}, x_{2} \in \mathbb{C}\left(\ell_{i_{1}}, \ldots, \ell_{i_{k}}\right)$. So, up to exchanging $x_{1}$ for $x_{2}$, the family $\left(x_{2}, \ell_{i_{1}}, \ldots, \ell_{i_{k}}\right)$ is free and $x_{1}$ belongs to $\mathbb{C}\left(x_{2}, \ell_{i_{1}}, \ldots, \ell_{i_{k}}\right)$. In other words, there exists an index $\kappa$ such that 
$\ell_{i_{\kappa}} \in \mathbb{C}\left(x_{1}, x_{2}, \ell_{i_{1}} \ldots, \check{\ell}_{i_{\kappa}}, \ldots, \ell_{i_{k}}\right)$. So, let $\ell_{i_{k+1}}, \ldots, \ell_{i_{n-1}}$ be other factors of $h$. From Remark $5.5,\left(x_{1}, x_{2}, \ell_{i_{1}}, \ldots, \check{\ell}_{i_{\kappa}}, \ldots, \ell_{i_{n-1}}\right)$ defines the origin and we have

$$
v=\sum_{j=k+1}^{n-1} \frac{v\left(\ell_{i_{j}}\right)}{\Delta^{x_{1}, x_{2}, \ell_{i_{1}}, \ldots, \check{\ell}_{i_{\kappa}}, \ldots, \check{\ell}_{i_{j}}, \ldots, \ell_{i_{n-1}}\left(\ell_{i_{j}}\right)}} \Delta^{x_{1}, x_{2}, \ell_{i_{1}}, \ldots, \check{\ell}_{i_{\kappa}}, \ldots, \check{\ell}_{i_{j}}, \ldots, \ell_{i_{n-1}}}
$$

since the two members are equal on $g, \ell_{i_{1}}, \ldots, \ell_{i_{n-1}}$. Hence we conclude exactly

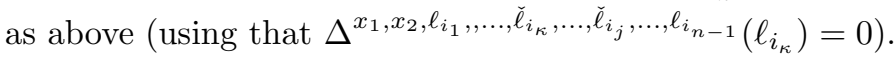

Of course, the inclusion $I \subset \operatorname{Ann}_{\mathcal{D}}(1 / h) g^{s}$ is clear. In order to get the reverse inclusion, we will just prove that $\operatorname{gr} \operatorname{Ann}_{\mathcal{D}}(1 / h) g^{s}$ is included in $\mathcal{I}$. Indeed, we conclude also easily by induction on order of operators in $I$, just as in the proof of Proposition 3.5.

Let us study $\operatorname{char}_{\mathcal{D}} \mathcal{D}(1 / h) g^{s} \subset T^{*} \mathbb{C}^{n}$, the characteristic variety of $\mathcal{D}(1 / h) g^{s}$. It is easy to check that $\mathcal{O} \subset \mathcal{D} 1 / h$ and that:

$$
\mathcal{O}\left[1 / \ell_{i_{1}} \cdots \ell_{i_{k}}\right]=\mathcal{D} 1 / \ell_{i_{1}} \cdots \ell_{i_{k}} \subset \mathcal{D} 1 / h
$$

for all $1 \leq i_{1}<\cdots<i_{k} \leq p, 1 \leq k \leq n-1$, under our assumption on $h$ (using that -1 is the only integral root of the Bernstein polynomial of a normal crossing). So the characteristic variety of $\mathcal{D} 1 / h$ contains the conormal bundles $T_{\mathbb{C}^{n}}^{*} \mathbb{C}^{n}$ and $T_{L_{i_{1}} \cap \cdots \cap L_{i_{k}}}^{*} \mathbb{C}^{n}, 1 \leq i_{1}<\cdots<i_{k} \leq p, k \leq n-1$, where $L_{i}=\operatorname{ker} \ell_{i} \subset \mathbb{C}^{n}$. Moreover, using Proposition 2.14.4 of [8], we deduce that $\operatorname{char}_{\mathcal{D}} \mathcal{D}(1 / h) g^{s}$ is the union of the subspaces $W_{g}$ and $W_{g \mid L_{i_{1}} \cap \cdots \cap L_{i_{k}}}$, where $W_{g \mid X} \subset T^{*} \mathbb{C}^{n}$ is the closure of $\left\{(x, \xi+\lambda \mathrm{d} g(x)) ; \lambda \in \mathbb{C},(x, \xi) \in T_{X}^{*} \mathbb{C}^{n}\right\}$ for any subanalytic space $X \subset \mathbb{C}^{n}$. The following result gives defining equations of the spaces $W_{g \mid L_{i_{1}} \cap \cdots \cap L_{i_{k}}}$.

Assertion 2. - Let $\ell_{i_{1}}, \ldots, \ell_{i_{k}} \in\left(\mathbb{C}^{n}\right)^{*}, 1 \leq k \leq n-1$, be some factors of $h$.

(i) If $k=n-1$, then $W_{g \mid L_{i_{1}} \cap \ldots \cap L_{i_{k}}}$ is defined by $\ell_{1}, \ldots, \ell_{n-1}$.

(ii) Assume that $k<n-1$. If $\left(x_{1}, x_{2}, \ell_{1}, \ldots, \ell_{i_{k}}\right)$ is a free family, then $W_{g \mid L_{i_{1}} \cap \cdots \cap L_{i_{k}}}$ is defined by $\ell_{1}, \ldots, \ell_{k}$, one nonzero element $\sigma\left(\Delta_{K}^{g, \ell_{1}, \ldots, \ell_{i_{k}}}\right)$, and the principal symbols of $n-k-2$ vector fields $\Delta_{K}^{x_{1}, x_{2}, \ell_{1}, \ldots, \ell_{i_{k}}}$ defining a free family.

(iii) Assume that $k<n-1$. If there exists an index $\kappa, 1 \leq \kappa \leq k$, such that $\ell_{i_{\kappa}} \in \mathbb{C}\left(x_{1}, x_{2}, \ell_{1}, \ldots, \check{\ell}_{i_{\kappa}}, \ldots, \ell_{i_{k}}\right)$, then $W_{g \mid L_{i_{1}} \cap \ldots \cap L_{i_{k}}}$ is defined by $\ell_{1}, \ldots, \ell_{k}$, and the principal symbols of $n-k-1$ vector fields $\Delta_{K}^{x_{1}, x_{2}, \ell_{1}, \ldots, \check{\ell}_{i_{\kappa}}, \ldots, \ell_{i_{k}}}$ defining a free family.

Proof. - In each case, it is easy to check that the $(n-1)$-given elements form a $\operatorname{gr}^{F} \mathcal{D}$-regular sequence and define an irreducible space in $T^{*} \mathbb{C}^{n}$. Moreover, they are zero on $W_{g \mid L_{i_{1}} \cap \cdots \cap L_{i_{k}}}$. So the assertion is clear, since $W_{g \mid L_{i_{1}} \cap \cdots \cap L_{i_{k}}}$ is irreducible of dimension $n+1$. 
So let $P \in \operatorname{Ann}_{\mathcal{D}}(1 / h) g^{s}$ be a nonzero operator of order $d$. Then $\sigma(P)$ is zero on $\operatorname{char}_{\mathcal{D}} \mathcal{D}(1 / h) g^{s}$. Let us prove that $\sigma(P) \in \mathcal{I}$. Using the inclusion $W_{g \mid L_{i_{1}} \cap \cdots \cap L_{i_{n-1}}} \subset \operatorname{char}_{\mathcal{D}} \mathcal{D}(1 / h) g^{s}$, we have $\sigma(P) \in\left(\ell_{i_{1}}, \ldots, \ell_{i_{n-1}}\right) \mathcal{O}[\xi]$ for $1 \leq i_{1}<\cdots<i_{n-1} \leq p$. Remark that:

$$
\bigcap_{1 \leq i_{1}<\cdots<i_{n-1} \leq p}\left(\ell_{i_{1}}, \ldots, \ell_{i_{n-1}}\right) \mathcal{O}=\sum_{1 \leq i_{1}<\cdots<i_{n-2} \leq p}\left[\prod_{i \neq i_{1}, \ldots, i_{n-2}}^{\left[\ell_{i}\right] \mathcal{O}}\right.
$$

(by induction on $p \geq n$, using that every sequence $\left(\ell_{i_{1}}, \ldots, \ell_{i_{n}}\right)$ is regular). Thus, we can write:

$$
\sigma(P)=\sum_{1 \leq i_{1}<\cdots<i_{n-2} \leq p} A_{i_{1}, \ldots, i_{n-2}}^{(0)}\left(\prod_{i \neq i_{1}, \ldots, i_{n-2}} \ell_{i}\right)
$$

for some $A_{i_{1}, \ldots, i_{n-2}}^{(0)} \in \mathcal{O}[\xi]$ zero or homogeneous of degree $d$.

Now let $\ell_{i_{1}}, \ldots, \ell_{i_{n-2}}$ be some factors of $h$ such that $\left(x_{1}, x_{2}, \ell_{i_{1}}, \ldots, \ell_{i_{n-2}}\right)$ is a free family. From the inclusion $W_{g \mid L_{i_{1}} \cap \cdots \cap L_{i_{n-2}}} \subset \operatorname{char}_{\mathcal{D}} \mathcal{D}(1 / h) g^{s}$ and Assertion 2, we have:

$$
\sigma(P) \in\left(\ell_{i_{1}}, \ldots, \ell_{i_{n-2}}, \sigma\left(\Delta^{g, \ell_{i_{1}}, \ldots, \ell_{i_{n-2}}}\right)\right) \mathcal{O}[\xi] .
$$

So, using that the sequences $\left(\ell_{i}, \ell_{i_{1}}, \ldots, \ell_{i_{n-2}}, \sigma\left(\Delta^{g, \ell_{i_{1}}, \ldots, \ell_{i_{n-2}}}\right)\right), i \neq i_{1}, \ldots, i_{n-2}$, are regular, we deduce: $A_{i_{1}, \ldots, i_{n-2}}^{(0)} \in\left(\ell_{i_{1}}, \ldots, \ell_{i_{n-2}}, \sigma\left(\Delta^{g, \ell_{i_{1}}, \ldots, \ell_{i_{n-2}}}\right)\right) \mathcal{O}[\xi]$. Remark that we get a similar result when $\left(x_{1}, x_{2}, \ell_{i_{1}}, \ldots, \ell_{i_{n-2}}\right)$ is not free. Hence $\sigma(P)$ may be written:

$$
\sigma(P)=U+\sum_{1 \leq i_{1}<\cdots<i_{n-3} \leq p} A_{i_{1}, \ldots, i_{n-3}}^{(1)}\left(\prod_{i \neq i_{1}, \ldots, i_{n-3}} \ell_{i}\right)
$$

where $A_{i_{1}, \ldots, i_{n-3}}^{(1)} \in \mathcal{O}[\xi]$ are zero or homogeneous of degree $d$, and $U \in \mathcal{I}$ (with the help of Assertion 1). Up to a division by $\mathcal{I}$, we will assume that $U=0$. Iterating this process with $W_{g \mid L_{i_{1}} \cap \cdots \cap L_{i_{k}}}, 1 \leq k \leq n-2$, we get $\sigma(P)-A^{(n-2)} h$ belongs to $\mathcal{I}$. Thus, using that $W_{g} \subset \operatorname{char}_{\mathcal{D}} \mathcal{D}(1 / h) g^{s}$, we have:

$$
A^{(n-2)} \in\left(\sigma\left(\Delta^{g}\right), \xi_{3}, \ldots, \xi_{n}\right) \mathcal{O}[\xi] .
$$

So $A^{(n-2)} h \in \mathcal{I}$, and we conclude that $\sigma(P) \in \mathcal{I}$. This ends the proof.

Proof of Theorem 5.2. - From Proposition 1.10, we have $\mathcal{D} 1 / h=\mathcal{O}[1 / h]$, and $(\mathcal{D} 1 / h)\left[1 / h^{\prime}\right]=\mathcal{O}\left[1 / h^{\prime} h\right]$ is generated by $1 / h^{\prime} h$. Thus, using Proposition 3.1 of [18], we deduce that $\operatorname{Ann}_{\mathcal{D}} 1 / h^{\prime} h$ is generated by $\sum_{i=1}^{n} x_{i}\left(\partial / \partial x_{i}\right)+p+q$ and the elements of $\operatorname{Ann}_{\mathcal{D}}(1 / h) h^{\prime s}$. We conclude with Proposition 5.4.

TOME $132-2004-\mathrm{N}^{\mathrm{O}} 4$ 


\section{BIBLIOGRAPHY}

[1] Artin (M.) - On the solution of analytic equations, Invent. Math., t. 5 (1968), pp. 277-291.

[2] BJÖrk (J.E.) - Analytic D-Modules and Applications, Kluwer Academic Publishers, 1993.

[3] Calderón-Moreno (F.J.) - Logarithmic differential operators and logarithmic de Rham complexes relative to a free divisor, Ann. Sci. École Norm. Sup., t. 32 (1999), pp. 577-595.

[4] Calderón-Moreno (F.J.) \& Narváez-Macarro (L.) - The module $\mathcal{D} f^{s}$ for locally quasi-homogeneous free divisors, Compositio Math., t. 134 (2002), pp. 59-74.

[5] Castro-Jiménez (F.J.), Mond (D.) \& Narváez-Macarro (L.) - Cohomology of the complement of a free divisor, Trans. Amer. Math. Soc., t. 348 (1996), pp. 3037-3049.

[6] Castro-Jiménez (F.J.) \& UCHA (J.M.) - Explicit comparison theorems for $\mathcal{D}$-modules, effective methods in rings of differential operators, J. Symb. Comput., t. 32 (2001), pp. 677-685.

[7] _ Free divisors and duality for $\mathcal{D}$-modules, Proc. Steklov Inst. Math., t. 238 (2002), pp. 97-105.

[8] Ginsburg (V.) - Characteristic varieties and vanishing cycles, Invent. Math., t. 84 (1986), pp. 327-402.

[9] Grothendieck (A.) - On the de Rham cohomology of algebraic varieties, Pub. Math. IHES, t. 29 (1966), pp. 95-105.

[10] Holland (M.) \& Mond (D.) - Logarithmic differential forms and the cohomology of the complement of a divisor, Math. Scand., t. 83 (1998), pp. 235-254.

[11] Kashiwara (M.) - B-functions and holonomic systems, Invent. Math., t. 38 (1976), pp. 33-53.

[12] Malgrange (B.) - Le polynôme de Bernstein d'une singularité isolée, Lecture Notes in Math., vol. 459, Springer-Verlag, 1975, pp. 98-119.

[13] Saito (K.) - Quasihomogene isolierte Singularitäten von Hyperflächen, Invent. Math., t. 14 (1971), pp. 123-142.

[14] - Theory of logarithmic differential forms and logarithmic vector fields, J. Fac. Sci. Univ. Tokyo, t. 27 (1980), pp. 265-291.

[15] - On microlocal b-function, Bull. Soc. Math. France, t. 122 (1994), pp. $163-184$.

[16] TORRELli (T.) - Équations fonctionnelles pour une fonction sur un espace singulier, Thèse, Université de Nice-Sophia Antipolis, 1998.

[17] _ Un calcul de polynôme Bernstein associé à un faisceau de coniques non dégénéré, C. R. Acad. Sci. Paris Sér. I Math., t. 331 (2000), pp. 47-50. 
[18] _ Polynômes de Bernstein associés à une fonction sur une intersection complète à singularité isolée, Ann. Inst. Fourier (Grenoble), t. 52 (2002), pp. 221-244.

[19] _ Sur les germes de fonctions méromorphes définis par un système différentiel d'ordre 1, prépublication 45/2002 de l'Institut Élie Cartan de Nancy, 2002.

[20] VArchenko (A.N.) - Asymptotic Hodge structure in the vanishing cohomology, Math. USSR-Izv., t. 18 (1982), pp. 469-512.

[21] Walther (U.) - Bernstein-Sato polynomial versus cohomology of the Milnor fiber for generic arrangements, Compositio Math., to appear; arXiv:math.AG/0204080.

[22] Wiens (J.) \& YuZvinsky (S.) - De Rham cohomology of logarithmic forms on arrangements of hyperplanes, Trans. Amer. Math. Soc., t. 349 (1997), pp. 1653-1662.

[23] Yano (T.) - On the theory of b-functions, Publ. RIMS Kyoto Univ., t. 14 (1978), pp. 111-202. 